\title{
Radial crystallization difference of melt spun polypropylene fiber along spinning line
}

Dan Pan ${ }^{\mathrm{a}, \mathrm{b}}$, Houkang $\mathrm{He}^{\mathrm{a}}$, Junfen Sun ${ }^{\mathrm{a}}$, Min Cao ${ }^{\mathrm{a}}$, Zongyi Qin ${ }^{\mathrm{a}}$, Long Chen ${ }^{\mathrm{a}}$

${ }^{a}$ State Key Laboratory for Modification of Chemical Fibers and Polymer Materials, College of Materials Science and Engineering, Donghua University, Shanghai 201620, China

${ }^{\mathrm{b}}$ Laboratory of Advanced Fibers, Empa, Swiss Federal Laboratories for Materials Science and Technology, Lerchenfeldstrasse 5, CH-9014 St. Gallen, Switzerland

\section{Corresponding authors:}

Long Chen, State Key Laboratory for Modification of Chemical Fibers and Polymer

Materials, College of Materials Science and Engineering, Donghua University,

Shanghai 201620, China.

Emails: happyjack@dhu.edu.cn.

Zongyi Qin, State Key Laboratory for Modification of Chemical Fibers and Polymer

Materials, College of Materials Science and Engineering, Donghua University,

Shanghai 201620, China.

Emails: phqin@dhu.edu.cn.

\section{ABSTRACT}

The radial crystallization difference of polypropylene (PP) fiber along spinning line was investigated via synchrotron radiation micro-beam X-ray diffraction analysis $(\mu-\mathrm{XRD})$ for the first time. Running fibers were collected at different spinning line position to study its radial crystallization difference. The distribution of crystallinity, 
crystal form and crystal size of PP fiber were obtained based on peak deconvolution. The relation between radial crystallization difference and processing condition was also investigated. The results indicate that the crystallinity of PP in the center region is found higher than the surface due to the radial temperature gradient during melt spinning, and the structure of fiber is highly sensitive to the radial temperature gradient. The crystallinity increased along spinning line and reach a steady rate after $50 \mathrm{~cm}$ of spinning line position. The crystallite size increased before $50 \mathrm{~cm}$ of spinning line position and has a slight decrease after $50 \mathrm{~cm}$ due to the growth and splitting of crystal. These results display a 2-D view of crystallization development of fiber during the melt spinning and give us a basic knowledge about the relation between structure evolution and processing conditions both in axis and radial direction.

\section{Introduction}

The manufacture of polymeric products is usually carried out by melting process which involves an interplay between fluid mechanics and heat transfer in rheological complex liquids. The final properties of polymer products depend on the micro- and macro-structure of polymers which are highly influenced by the molecular structure and melt processing conditions. Small differences in the structure of a polymer chain aggregation or morphology will result in huge differences in the performance of the products. Normally, the temperature, stress rate and strain profile is not uniform in most of melt processing, ${ }^{1,2}$ such as the parabolic shear profile in extrusion die flow and non-isothermal elongation during melt spinning. These processing history differences in very small domain result in the variety of micro- and macro- structure 
of fibers. For instance, the formation of skin-core structure in the melt spinning fibers due to the gradient temperature and elongation force along the radial direction. ${ }^{3}$ Therefore, the clarification of the structure difference and its development during melt spinning of fiber could help to better control the structure and properties of fiber materials via tuning the processing parameters.

It is known that there is an non-uniform distribution of temperature along the radial direction in melt spinning of fibers due to the heat transfer from the molten fiber to surrounding medium. ${ }^{4}$ The radial temperature difference in turns affects the radial viscosity and stress fields in the running fiber, which can generate a hierarchical structure like skin-core structure. ${ }^{3,5}$ Furthermore, the temperature difference also influences the micro-rheological behavior of the second phase in polymer blend fibers. ${ }^{4,6,7}$ It was found that the second dispersed phase in the center region with higher temperature has better deformability than that in the surface region during the melt spinning of blend fibers, which could generate gradient dispersed morphology in blend fibers. Most of the researches in this area are focused on exploring the molecular structure and morphology development of fibers along the spinning line during melt spinning, such as the mechanism of nucleation and growth of crystal, the orientation of both lamellar stacks and crystallites and the structure change during the early stages of crystallization, via the on-line X-ray scattering technique. ${ }^{8-16}$

However, the in-situ X-ray scattering technique hasn't been used to detect the radial structure difference of fiber during melt spinning due to the shaking of running filament and size of the beam line make it difficult to focus on the running fibers as 
far as we know. Still there are some ways to investigate the radial structure differences in fibers. Matsuo and Kase ${ }^{17}$ used a numerical method to calculate the radial temperature distribution of fiber and found that the radial temperature difference leads to a radial difference in the birefringence, density by the means of dye experiment and optical microscopy. With the development of synchrotron radiation micro-beam X-ray diffraction technique, beam line is minimized to micrometer size which can support microscopic position resolution to detect the information of micro-structure at different radial position of fiber. Muller et. al. ${ }^{18}$ use X-ray microbeam which perpendicular to a viscose fiber axis to scan the fiber surface from edge to centre. Muller's study showed there is no significant variation of crystallinity from skin to core, while the cellulose molecules in the core region are much less oriented than that in the surface part of the fiber. Kobayashi et. al. ${ }^{19}$ also used a similar method as Muller's to detect the crystalline strain under tension both in inner-surface and core part of carbon fiber and evaluate the heterogeneous stress distribution along fiber cross section. However, the X-ray microbeam diffraction pattern overlap surface and central diffraction patterns of fiber, which would interfere the characterization of skin/core difference. The on-line quantitative characterization of structure or properties with respect to radial distribution in fiber is still tough due to the setup for this purpose hasn't been developed so far.

In this study, PP fibers were melt-spun and collected at different spinning line position. The method for the preparation of fiber section is emphasized. Synchrotron radiation X-ray micro-beam diffraction was employed to scanning the PP fiber 
sections at different radial position to study the radial structure difference. Radial crystallization distribution of PP fiber at the spinning line was obtained and gives us a basic knowledge about the relation between structure evolution and processing conditions both in axis and radial direction, which could support further studies in this field.

\section{EXPERIMENTS}

\section{Materials}

Isotactic polypropylene (PP) used in this work was supplied by Donghua University. The melt flow index of PP is $46.0 \mathrm{~g} / 10 \mathrm{~min}\left(230^{\circ} \mathrm{C} / 2.16 \mathrm{~kg}-\mathrm{ASTM}\right.$ Method D 1238). The number average molecular weight and the polymer dispersity index of PP is $75 \mathrm{~kg} \mathrm{~mol}^{-1}$ and 4.1 respectively. $\mathrm{PP}$ was dried at $80{ }^{\circ} \mathrm{C}$ in a vacuum oven for $12 \mathrm{~h}$ before any use.

\section{Melt spinning}

PP fibers were melt-spun on a lab-scale melt spinner with a single screw extruder ( $D=25 \mathrm{~mm}, \mathrm{~L} / \mathrm{D}=28)$. The running filaments were quenched in air and taken up by 500 $\mathrm{m} / \mathrm{min}$. The main spinning parameters are summarized in Table 1. A capturing device was fixed in spinning shaft to collect running fibers at different position of spinning line. The capturing device consists of a trigger-type knife, a support frame with slide rail and vertical position scale. This trigger-type knife consists of a sharp blade and a dull blade is fixed in the support frame. The dull blade located on the top of knife acts as sample clamper to fix the captured fibers. The distance between the dull blade and sharp blade is $10 \mathrm{~cm}$. The detail about the capturing device is shown in our previous 
study. ${ }^{6}$ The captured samples were taken out and thrown into liquid nitrogen immediately to freeze its structure.

Table 1 Parameters in melt spinning

\begin{tabular}{ccc}
\hline Data & Unit & Value \\
\hline Head pressure of extruder & $\mathrm{MPa}$ & $7 \pm 0.2$ \\
Number of spinneret orifice & - & 24 \\
Diameter of spinneret orifice & $\mathrm{mm}$ & 0.3 \\
L/D of spinneret orifice & - & 2.5 \\
Barrel temperatures of extruder & ${ }^{\circ} \mathrm{C}$ & $200 / 205 / 205$ \\
Temperature of spinneret & ${ }^{\circ} \mathrm{C}$ & 205 \\
Flow rate in volume & $\mathrm{cm}^{3} \mathrm{~min}^{-1}$ & 27 \\
Velocity of cross air & $\mathrm{m} \mathrm{s}^{-1}$ & 0.1 \\
Temperature of cross air & ${ }^{\circ} \mathrm{C}$ & 25 \\
Start point of cross air from spinneret & $\mathrm{m}$ & 0.1 \\
End point of cross air from spinneret & $\mathrm{m}$ & 1.5 \\
Distance from spinneret to the first roller & $\mathrm{m}$ & 1.5 \\
\hline
\end{tabular}

Thermal analysis

The differential scanning calorimetry (DSC Q2000, TA instrument) experiments were carried out to obtain the thermal behavior during melt process. The temperature was calibrated with indium $\left(156.6{ }^{\circ} \mathrm{C}\right)$. About 3-5 mg PP fiber sample sealed in a tzero aluminum pan. The temperature procedure running from 30 to $230{ }^{\circ} \mathrm{C}$ at a rate of 10 ${ }^{\circ} \mathrm{C} / \mathrm{min}$ under a nitrogen flow rate of $50 \mathrm{ml} / \mathrm{min}$.

The crystallinity $\mathrm{X}_{c}$ of PP fiber was determined as

$\mathrm{X}_{c}=\frac{\Delta H_{m}-\Delta H_{c c}}{\Delta H_{f}^{0}} \times 100$

where $\Delta H_{m}$ is the melting enthalpy of PP fiber sample, $\Delta H_{c c}$ the cold crystallization enthalpy, $\Delta H_{f}^{0}$ is the heat fusing of $100 \%$ crystalline PP.X-ray diffraction

To prepare fiber sections for the X-ray microbeam diffraction, the collected fibers were combed carefully and embedded in Spurr's epoxy resin. The detail of the 
embedding process is provided in previous work. ${ }^{20}$ A Leica RM2126 RT rotary microtome (Leica Microsystems, Germany) was used to slice the embedded fibers along the fiber axis to obtain $6 \mu \mathrm{m}$ thickness of fiber sections at $-10{ }^{\circ} \mathrm{C}$. Then these fiber sections were carefully adhered in polyimide film tape $(5413,3 \mathrm{M})$.

The X-ray microbeam diffraction experiment was performed at the hard X-ray micro-focus beam-line BL15U in Shanghai Synchrotron Radiation Facility (SSRF). ${ }^{21}$ The polyimide film tape with fiber sections on its surface was fixed in a foursquare holder and placed on a high precision 7-axis rotary stage. A monochromatic beam of wavelength $\lambda=0.124 \mathrm{~nm}$ was obtained by a monochromator. The beam diameter at the sample position was collimated to be about $2.2 \mu \mathrm{m} \times 2.0 \mu \mathrm{m}$ (horizontal $\times$ vertical), which was determined by the knife-edge technique using a thin tantalum blade. The energy was set at $10 \mathrm{keV}$. The distance between the sample and detector is $172 \mathrm{~mm}$. The sample location for the measurement was precisely determined using a microscope. The angle between microscope objective and fiber section is $45^{\circ}$. The section was placed in the center of the microscope view. The incident X-ray microbeam was introduced and mapping the specimen from upside of fiber section to the downside as shown in Figure 1 and Figure 2. The step length is set as $2 \mu \mathrm{m}$ and the exposing time is $10 \mathrm{~s}$. A background picture from pure polyimide film tape was also collected and subtracted from the diffraction pattern. The X-ray measurement data analysis (distance calibration, background correction, radial and azimuthal integration) was carried out using the Fit2d software package. ${ }^{22}$ 

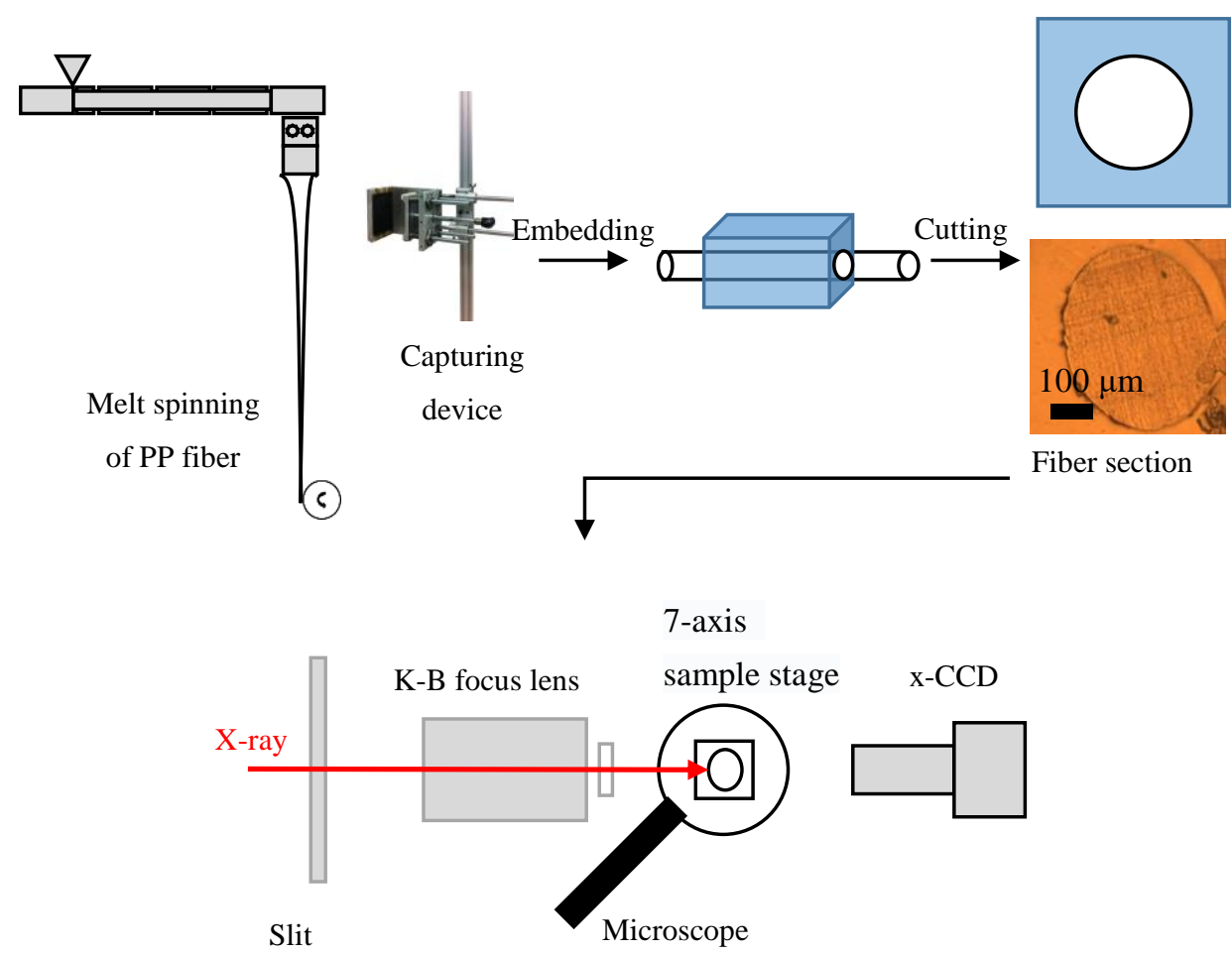

Figure 1. $\mu$-XRD mapping detection of PP fiber cross section.

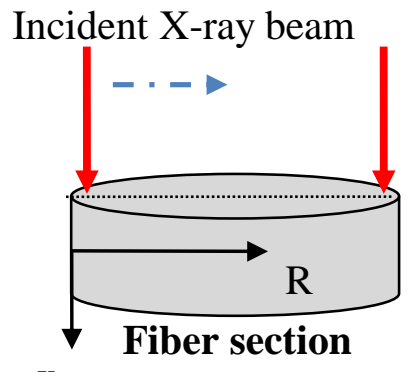

$\mathrm{X}$

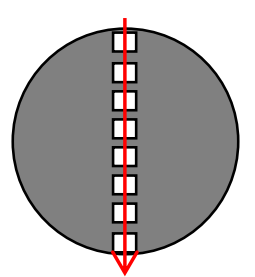

(b) Top view

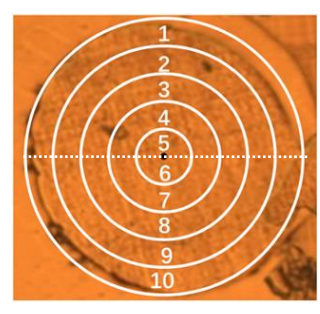

(c) Partition

Figure 2. Schematic instruction of $\mu$-XRD mapping detection (a) Side view of fiber section; (b) Top view of fiber section; (c) Partition of fiber section.

A Bruker® D8 Advance X-ray diffractometer system utilizing nickel filtered $\mathrm{CuK} \alpha$ radiation (wavelength of $1.54 \AA$ ) was also used to obtain the wide-angle X-ray diffraction profile of PP fiber. The voltage and current were set as $40 \mathrm{kV}$ and $40 \mathrm{~mA}$, respectively. 


\section{RESULTS AND DISCUSSION}

\section{$X$-ray microdiffraction}

The appearance of the diffraction pattern can reflect the nature of the crystalline phases in the specimen, such as crystallinity, size and orientation of crystal.

Figure 3 shows a typical 2-D wide angle X-ray diffraction pattern of PP fiber section collected at spinning position of $150 \mathrm{~cm}$ from spinneret. Since the small thickness of the PP fiber section the exposing time can't be too high to avoid structural damage of polymer from high flux X-ray, the detected X-ray diffraction signal of PP fiber section was very weak. It can be seen there are just two weak diffraction rings in the pattern after subtracting the background pattern in Figure 3. On the other hand, only diffraction rings can be seen in $2 \mathrm{D}$ diffraction pattern of semi-crystalline PP fiber section may indicates crystals are randomly distributed in the radial direction of fiber. The WAXD intensity profile at different detecting positions of fiber section obtained by integrating the whole azimuthal angle range of 2-D patterns are shown in Figure 4. The WAXD intensity near the fiber surface is relatively lower than that in the center of the fiber section. As indicated by these radial WAXD intensity profiles, there is a crystallinity gradient in the PP fiber section along the radial direction of PP fiber. Furthermore, the difference and development radial crystallization during melt spinning can be evaluated by analyzing the radial WAXD intensity profiles along spinning line. 


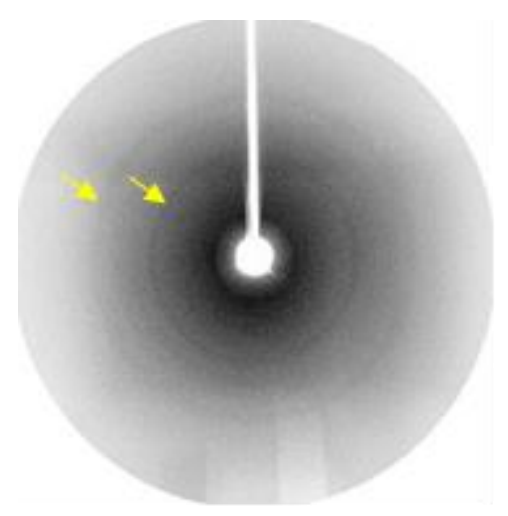

Figure 3. 2-D XRD pattern of take up fiber at spinning line position of $150 \mathrm{~cm}$

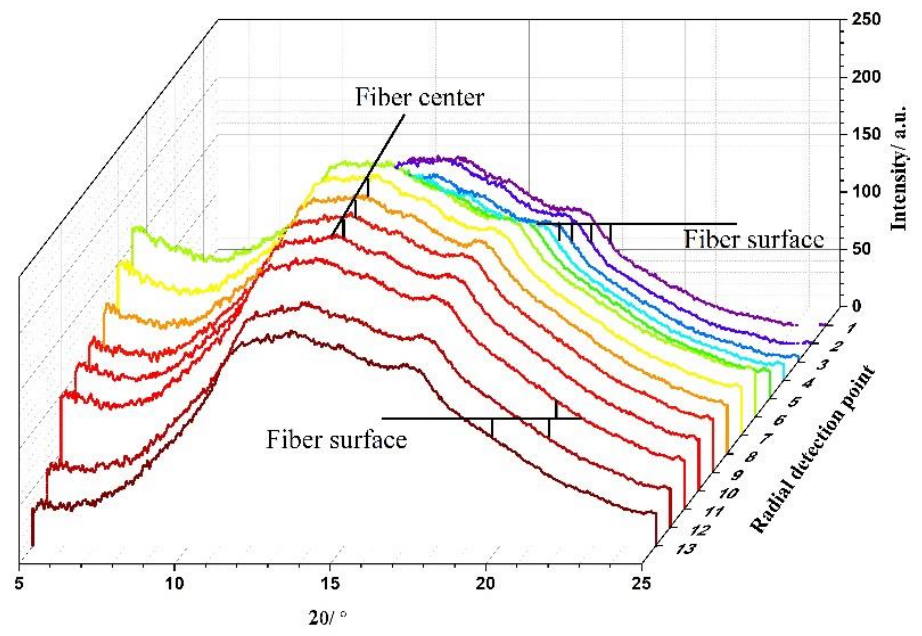

Figure 4. WAXD intensity profiles obtained from 2-D patterns at different radial position along radial direction of PP fiber.

\section{2-D distribution of crystallinity}

WAXD profiles showed in Figure 4 are very broad and diffused with only two identifiable diffraction peaks. So a peak deconvolution package ${ }^{22}$ was used to resolve the peak position of PP fiber X-ray diffraction spectra and to support the structure information for analysis. A Gaussian function was chosen to fit the X-ray diffraction peak profile. Both the amorphous and crystalline peak components of the WAXD intensity were dissolved as shown in Figure 5. In the WAXD profile, the reflections at $2 \theta=12.2^{\circ}, 17.1^{\circ}$ and $18.4^{\circ}$ are corresponding to the $\alpha$-form crystal reflections of 
(110), (040) and (130) planes respectively. While the (300) reflection at about $15.2^{\circ}$ and the (400) reflection at $20.4^{\circ}$ are the principal reflections of the $\beta$-crystals. ${ }^{23}$

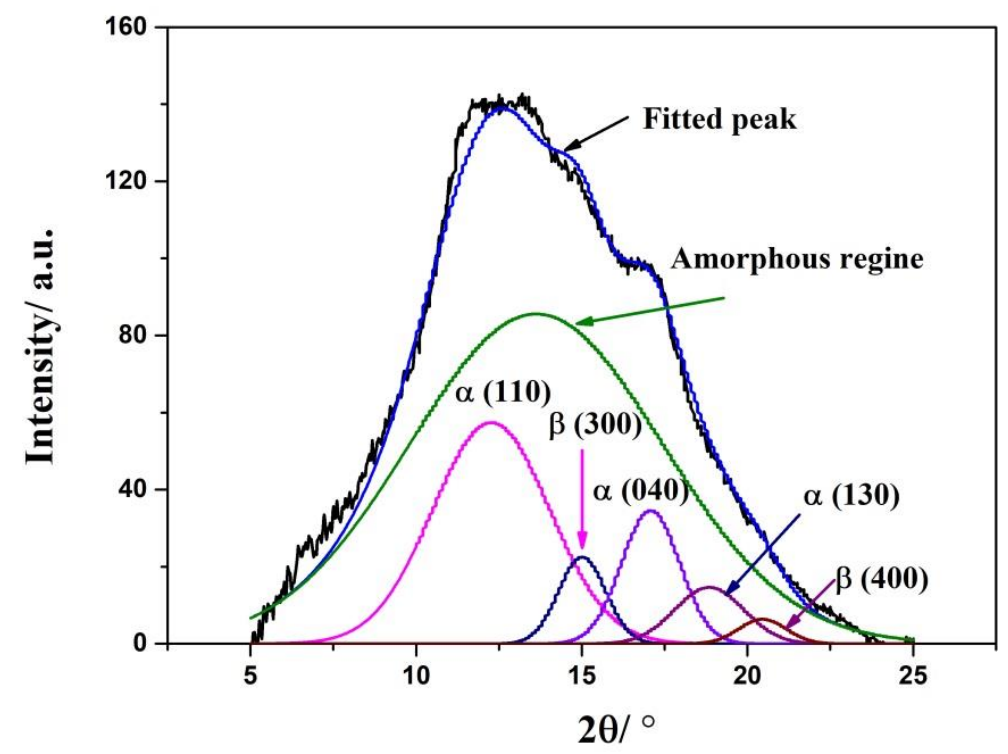

Figure 5. Deconvolution of WAXD profile

After fitting the curve of several crystalline and amorphous peaks, the crystallinity was determined as:

$$
\mathrm{X}_{c}=\frac{\sum A_{c}}{\sum A_{a}+\sum A_{c}}
$$

where $A_{c}$ is the integrated area of the crystalline reflection, $A_{a}$ the integrated area of the amorphous reflection.

The d-spacing was determined by Bragg equation,

$$
2 d \sin (\theta)=\mathrm{n} \lambda
$$

where $\mathrm{n}$ is the positive integer, $\theta$ the Bragg angle for the reflection concerned and $\lambda$ the wavelength of the incident X-ray beam.

The fiber section was partitioned into 10 radial zones with same interval distance to evaluate the radial structure difference in fiber sections as shown in Figure 2(c). Figure 6 shows the radial crystallinity profile of PP fiber collected at different 
spinning line positions. The crystallinity of PP in each radial zone was taken as the average crystallinity value of detecting points located in corresponding radial partition. The approximate number of detecting points for each fiber section is about 15-30. The results show that there is a radial difference in the crystallization between the center and surface region of polypropylene fiber. The crystallinity of PP fiber in the center region is higher than that in the surface and the maximum position of the crystallinity is off the fiber center. The radial crystallinity distribution was fitted by a Gauss function. The radial crystallinity distribution along the mapping direction was given based on the regression function and the assumed 2-D radial crystallinity distribution of PP fiber which has ten axial symmetry rings were also given in Figure 7.

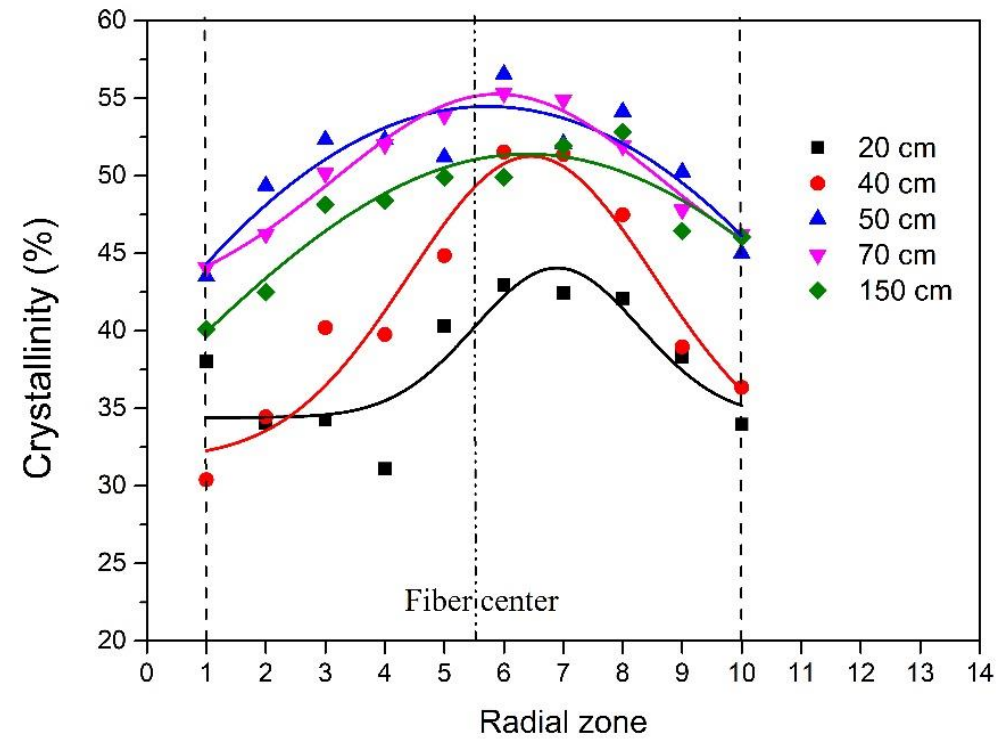

Figure 6. Radial crystallinity distribution of PP fiber collected at different spinning line position

As we know, polymer crystallization is a temperature and time depended process. The radial temperature gradient will induce different crystallization behavior of PP along radial direction during melt spinning. Figure 6 and Figure 7 show that there is higher crystallinity in the center of fiber which means higher temperature in the center 
region gives polymer chains more freedom and more time to fold into a crystal once the nucleation event happened during melt spinning. The radial temperature gradient leads to complex thermal-mechanical conditions during melt spinning and the variation of radial temperature distribution could also result in a radial structure difference. A quench air blow was applied to one side of the running fiber during melt spinning, which seems to result in an asymmetric distribution of crystallinity along the radial direction of fiber as shown in Figure 7. It also can be seen that the maximum crystallinity point tends to move to the center of the fiber section from Figure 7. These phenomena show by the sensitive of radial structure difference to the radial temperature difference. However, it should be note that we can't get an exact conclusion about how radial crystallization difference developed and the real radial crystallinity distribution during melt spinning of PP fiber due to the angle between detecting direction and air flow direction is not sure for each fiber section. 


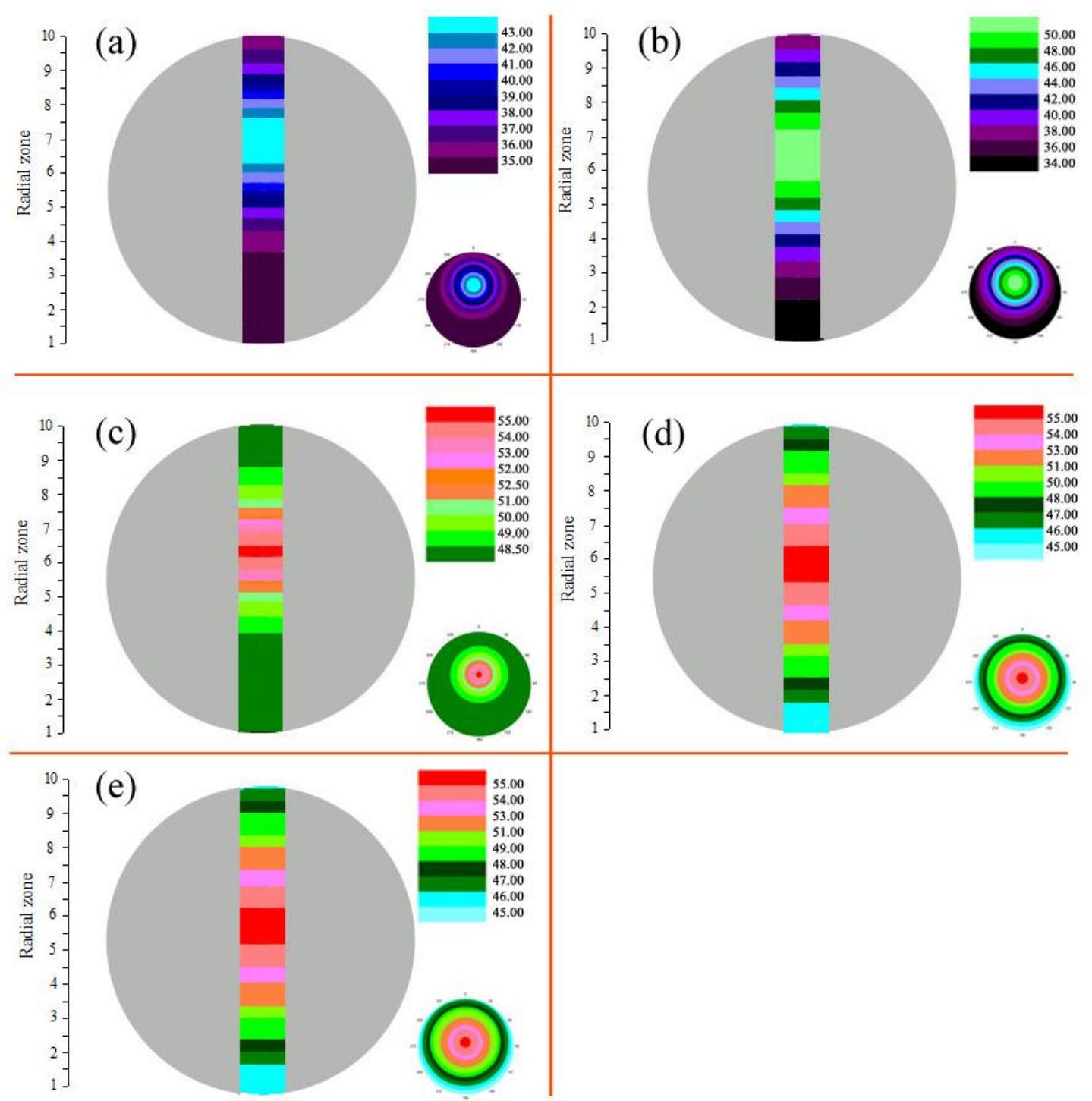

Figure 7. Radial crystallinity distribution along mapping direction in cross section of fiber collected at different spinning line position and the assumed 2-D radial crystallinity distribution as shown in the lower right corner of figure, (a) $20 \mathrm{~cm}$; (b) $40 \mathrm{~cm}$; (c) $50 \mathrm{~cm}$, (d) $70 \mathrm{~cm}$; (e) $150 \mathrm{~cm}$ 


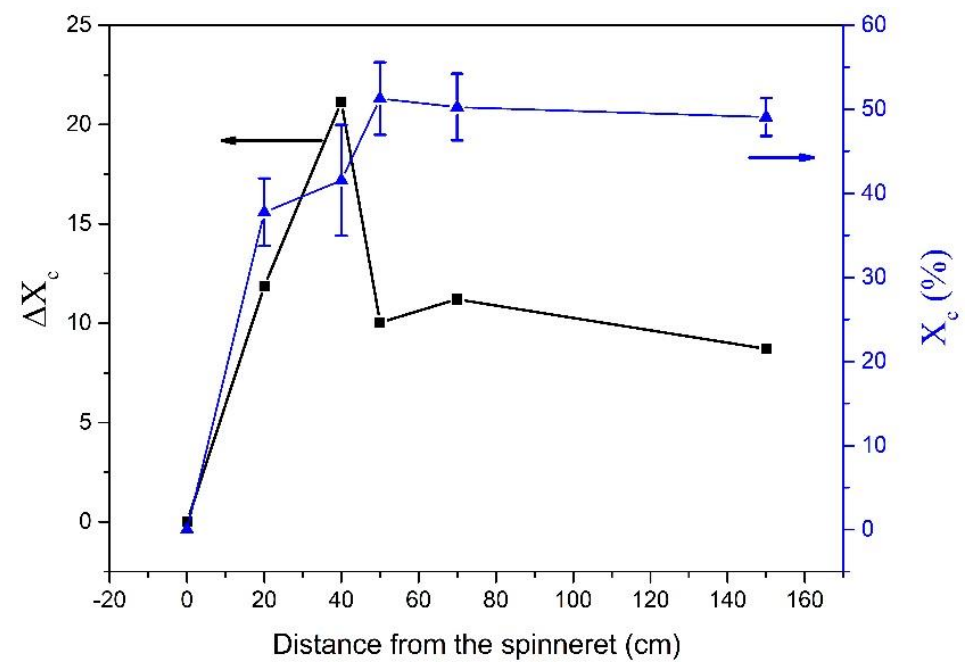

Figure 8. Crystallinity and $\Delta X_{c}$ of PP fiber along spinning line

Table 2. crystallinity of PP fiber determined from different methods

\begin{tabular}{cccc}
\hline & DSC & XRD & X-ray micro-focus beam-line \\
\hline$X_{c},(\%)$ & 42.00 & 47.94 & 48.24 \\
\hline
\end{tabular}

The average degree of crystallinity of PP and the maximum radial crystallinity difference $\Delta X_{c}$ at different spinning line position were plotted in Figure 8. The crystallinity difference $\Delta X_{c}$ is defined as the difference value between the highest and lowest crystallinity. The value of crystallinity and $\Delta X_{c}$ of PP fiber were assumed as 0 at $0 \mathrm{~cm}$ due to the temperature of the polymer melt at this position is higher than the melting temperature of PP. Figure 8 shows that the degree of crystallinity increased rapidly before spinning line position of $40 \mathrm{~cm}$ and then reached a stable value in our case. These results are corresponding to the studies of Kolb et.al ${ }^{9}$ and Lopes et.al ${ }^{10}$. The crystallinity of PP fiber (at the spinning line position of $150 \mathrm{~cm}$ ) is about $48.24 \%$. Table 2 shows the crystallinity of PP fiber obtained from DSC and x-ray diffraction analysis. the details about the DSC thermal properties and WAXD profile can be 
found in supporting information. Determined $X_{c}$ of PP fiber from different methods does not show dramatic differences between each other, which corroborate the data obtained from micro-beam X-ray diffraction technique. It can also be seen that $\Delta X_{c}$ increased along spinning line and reached the maximum value of $21.14 \%$ at the distance of $40 \mathrm{~cm}$ from the spinneret, then $\Delta X_{c}$ decreased along the spinning line. The profile of $\Delta X_{c}$ versus distance from the spinneret is coincided to the profile of radial temperature gradient versus distance from spinneret which is similar to those in our previous study ${ }^{2}$ where the radial temperature gradient has the maximum value at the position of about $40 \mathrm{~cm}$ at the take up speed of $500 \mathrm{~m} / \mathrm{min}$. This confirms that the radial distribution of crystallinity of polypropylene is depended on the radial temperature gradient of fiber during melt spinning.

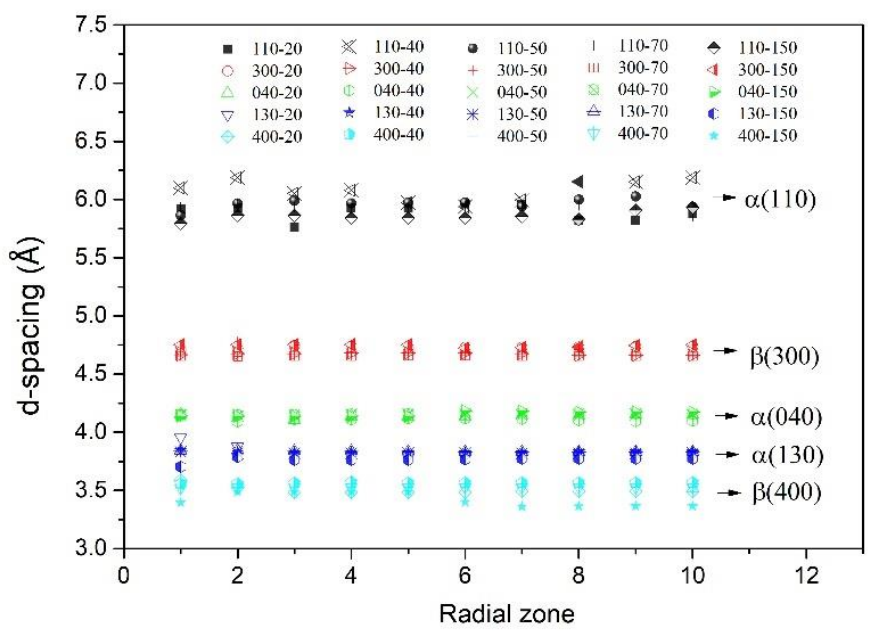

Figure 9. d-spacing of PP fiber of PP fiber collected at different spinning line position

Figure 9 shows the calculated d-spacings for the $\alpha(110), \alpha(040), \alpha(130) \beta(300)$ and $\beta(400)$ planes. The d-spacing variations for each plane is very small, which indicates a small variation in crystalline density along radial direction. Many experimental observations ${ }^{23,24}$ concluded that $\alpha$ crystalline form is much more 
thermodynamically stable than $\beta$ form. The $\beta$ crystals have many performance characteristics such as improved elongation at break and impact strength. The phase contents of $\alpha$ and $\beta$ crystalline phase were calculated by dividing integrated area of $\alpha$ or $\beta$ crystalline phase by integrated area of whole XRD profile and the development of phase contents of $\alpha$ and $\beta$ crystalline phase along the spinning line are shown in Figure 10. The phase contents of $\alpha$ and $\beta$ crystalline phase at $0 \mathrm{~cm}$ from the spinneret were assumed as $0 \%$. The content of $\alpha$ crystalline phase is much higher than $\beta$ form as expected, and both $\alpha$ and $\beta$ crystal contents level off after $50 \mathrm{~cm}$ of spinning line position, which shows similar trend of the development of crystallinity as shown in Figure 8 . However, there is still a slight change of crystalline phase content at the spinning position of $50 \mathrm{~cm}$, which may indicate some transformation between $\alpha$ and $\beta$ form at high elongational stress rate. ${ }^{25}$ But this transfer is not maintained after $50 \mathrm{~cm}$, which may indicate another transformation or the crystal distortion in this stage. Meanwhile, the asymmetric distribution of crystallinity across the fiber section could also lead to this small change in crystalline phase due to our mapping detection as carried out at one single direction. Figure 11 shows the radial distribution of $\alpha$ and $\beta$ crystalline phase content at different spinning position. One could see a similar radial distribution of crystallinity as shown in Figure 6. It indicates that the radial temperature gradient has a similar effect on the formation of $\alpha$ and $\beta$ crystalline phase in PP during melt spinning. 


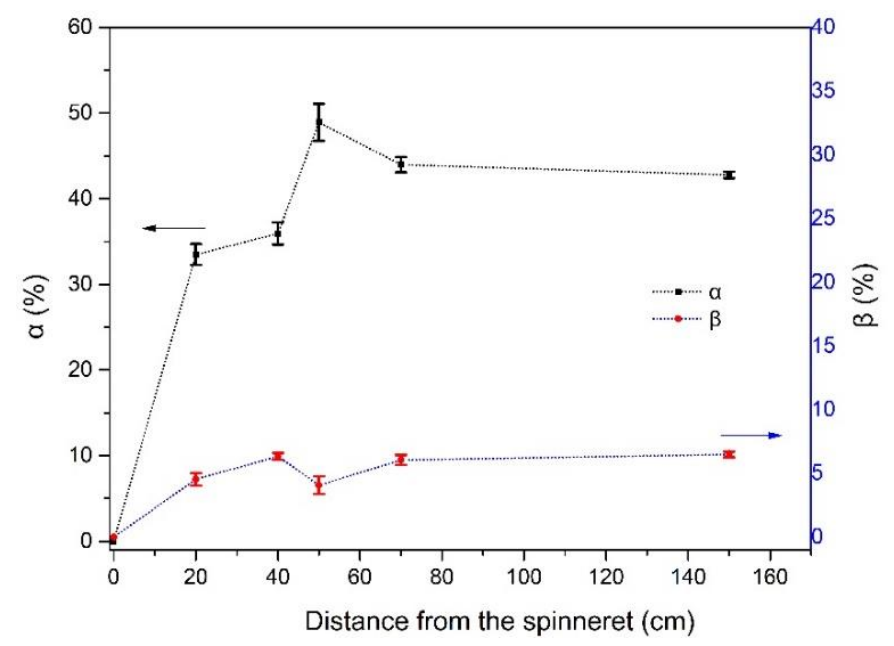

Figure 10. Crystalline phases content of $\alpha$ and $\beta$ form with error bar at different spinning line position

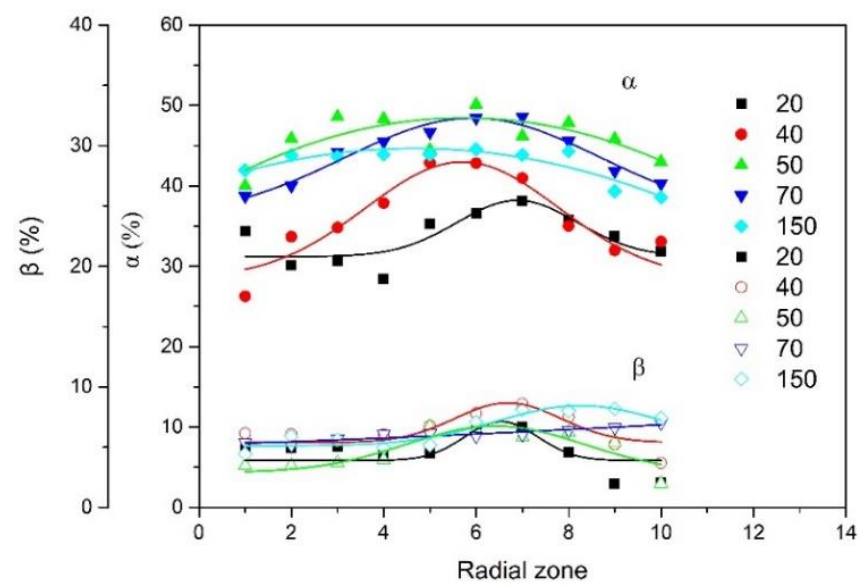

Figure 11. Radial distribution of crystalline phases content at different spinning line position

The crystal size was calculated from Scherrer equation, ${ }^{26}$

$$
L_{h k l}=\frac{k \lambda}{\beta \cos \theta}
$$

where $L_{h k l}$ is the mean length of the crystallite perpendicular to the (hkl) planes, $k$ is the a Scherrer factor usually taken as 0.89 for half-height widths, $\beta$ is the breadth at half maximum intensity in radians.

Crystallite sizes referred to two $\alpha 110, \alpha 040$ lattice planes were determined from the Scherrer equation based on half-height widths and $2 \theta$. The crystallite size of these two lattice planes at different spinning line position are shown in Figure 12. There is a rapid increase in the crystallite size before $50 \mathrm{~cm}$ of spinning line position and a slight 
decrease after $50 \mathrm{~cm}$. The rapid increase of the crystallite size comes from the rapid growth of polymer crystal in an appropriate temperature before $50 \mathrm{~cm}$. The decrease of crystallite size could contribute to the growth and splitting of crystal during extension process. $^{27}$ Splitting of crystal will happen under high spinning tension results from increasing take up velocity along the spinning line.

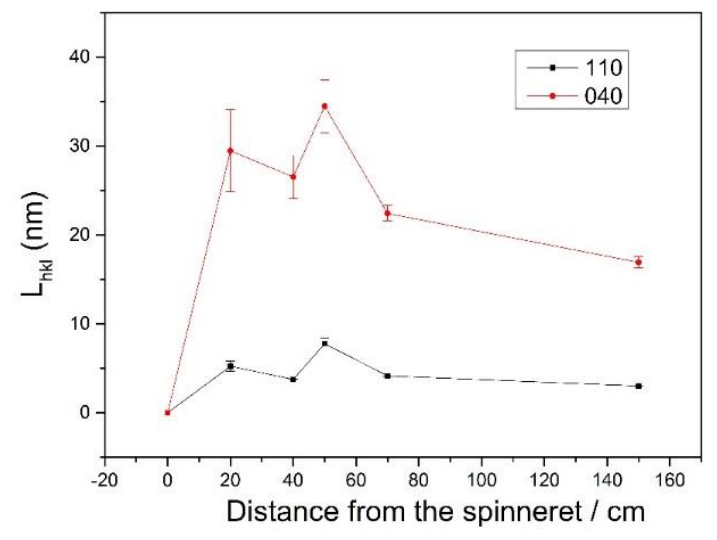

Figure 12. Crystallite size along spinning line position

\section{CONCLUSIONS}

A method for analysis of aggregation structure difference along radial direction of fiber was established based on $\mu$-XRD mapping and fine slice techniques. The radial crystallization difference of PP fiber was quantitatively investigated. The crystallinity of polypropylene in the center region is found higher than that of the surface, which shows us a crystallinity gradient morphology in pure polymer. There is a huge structure variation before $70 \mathrm{~cm}$ of spinning line position in the discussed case due to the variable temperature field. The radial crystallinity difference increased before $40 \mathrm{~cm}$ of spinning line position and decreased after $40 \mathrm{~cm}$, which is consistent with the development trend of the radial temperature gradient. The crystallite size of PP increased before $50 \mathrm{~cm}$ of spinning line position and have a slight decrease after 
$50 \mathrm{~cm}$ due to the growth and splitting of crystal result from the increasing spinning force during melt spinning process. These results give us a simple view of how aggregation structure developed both in radial and axis direction and how radial temperature distribution influence the crystallization of polypropylene during melt spinning. But more efforts, such as improving the online sample capturing process, obtaining the orientation information of molecular or crystal, are needed to clarify a more clearer view of radial aggregation structure difference development and to reveal the relationship between processing conditions and structure during melt spinning.

\section{ACKNOWLEDGEMENTS}

The authors thank the National Key Research and Development Program of China (2016YFB0302602) and the China Scholarship Council for financial support, and Shanghai Synchrotron Radiation Facility (SSRF) for technical support. The authors also thank Dr. Felix Reifler and Dr. Rudolf Hufenus from Empa for their kind help and suggestions.

\section{REFERENCES}

1. Yang, J.; White, J. L.; Jiang, Q., Polymer Engineering \& Science. 2010, 50, 1969-1977.

2. Chen, L.; He, H.; Zhang, Y.; Chen, Y.; Zhu, M., Fibers and Polymers. 2015, $16,449-462$.

3. Shimizu, J.; Okui, N.; Kikutani, T., Sen'i Gakkaishi. 1981, 37, T135-T142.

4. Pan, D.; Chen, L.; He, H.; Deng, K.; Qin, Z., Fibers and Polymers. 2016, 17, 1343-1351.

5. Konda, A.; Toriumi, K.; Nakajima, T., Sen'i Gakkaishi. 1985, 41, T530-T538.

6. He, H.; Chen, L.; Zhang, Y.; Hong, S.; Zhou, Y.; Zhu, M., Fibers and Polymers. 2014, 15, 1941-1949.

7. Xing, Q.; Zhu, M.; Wang, Y.; Chen, Y.; Zhang, Y.; Pionteck, J.; Adler, H. J., Polymer. 2005, 46, 5406-5416.

8. Ellison, M. S.; Lopes, P. E.; Pennington, W. T., J Eng Fiber Fabr. 2008, 3, 
$10-21$.

9. Kolb, R.; Seifert, S.; Stribeck, N.; Zachmann, H. G., Polymer. 2000, 41, 1497-1505.

10. Lopes, P. E.; Ellison, M. S.; Pennington, W. T., Plastics, Rubber and Composites. 2013, 35, 294-300.

11. Samon, J. M.; Schultz, J. M.; Hsiao, B. S., Polymer. 2002, 43, 1873-1875.

12. Samon, J. M.; Schultz, J. M.; Hsiao, B. S.; Khot, S.; Johnson, H. R., Polymer. 2001, 42, 1547-1559.

13. Samon, J. M.; Schultz, J. M.; Wu, J.; Hsiao, B.; Yeh, F.; Kolb, R., J Polym Sci Pol Phys. 1999, 37, 1277-1287.

14. Schultz, J. M.; Hsiao, B. S.; Samon, J. M., Polymer. 2000, 41, 8887-8895.

15. Cakmak, M.; Teitge, A.; Zachmann, H. G.; White, J. L., Journal of Polymer Science Part B: Polymer Physics. 1993, 31, 371-381.

16. Katayama, K.; Nakamura, K.; Amano, T., Kolloid-Zeitschrift und Zeitschrift für Polymere. 1968, 226, 125-134.

17. Matsuo, T.; Kase, S., Sen'i Gakkaishi. 1968, 24, 512-523.

18. Muller, M.; Riekel, C.; Vuong, R.; Chanzy, H., Polymer. 2000, 41, 2627-2632.

19. Kobayashi, T.; Sumiya, K.; Fujii, Y.; Fujie, M.; Takahagi, T.; Tashiro, K., Carbon. 2013, 53, 29-37.

20. He, H.; Chen, L.; Sun, S.; Wang, T.; Zhang, Y.; Zhu, M., Fibers and Polymers. 2014, 15, 744-752.

21. Zhang, L.; Yan, S.; Jiang, S.; Yang, K.; wang, H.; He, S.; Liang, D.; Zhang, L.; He, Y.; Lan, X., Nucler Science and Techniques,. 2015, 26, 1-8.

22. Hammersley, A. P.; Svensson, S. O.; Thompson, A., Nucl Instrum Meth A. 1994, 346, 312-321.

23. Takahashi, T., Sen'i Gakkaishi. 2002, 58, 357-364.

24. Yang, S.-G.; Chen, Y.-H.; Deng, B.-W.; Lei, J.; Li, L.; Li, Z.-M., Macromolecules. 2017, 50, 4807-4816.

25. Nadella, H. P.; Spruiell, J. E.; White, J. L., Journal of Applied Polymer Science. 1978, 22, 3121-3133.

26. Patterson, A. L., Physical Review. 1939, 56, 978-982.

27. Somani, R. H.; Yang, L.; Zhu, L.; Hsiao, B. S., Polymer. 2005, 46, $8587-8623$. 